\title{
Alterações oculares na hanseníase, observadas em pacientes ambulatoriais do serviço de referência da cidade de Rio Branco, Acre - Brasil
}

\author{
Eye lesions observed in outpatients of a leprosy referencecenter at Rio Branco, \\ stateof Acre, Brazil
}

\author{
Renaldo Duarte Moreno ${ }^{1}$ \\ William Woods ${ }^{2}$ \\ Natália Moreno $^{3}$ \\ Ronald Trindade ${ }^{4}$ \\ JoséTavares-Neto ${ }^{5}$
}

Apoio: PET- Medicina (UFBA); CNPq

Professor-assistente, Mestre, Curso de Medicina da Universidade Federal do Acre; Médico do Serviço de Oftalmologia da Fundação Hospital do Estado do Acre. Médico do Serviço de Dermatologia Sanitária da Secretaria de Saúde do Estado do Acre - SESACRE.

Acadêmica da Faculdade de Medicina de Jundiaí - SP.

${ }^{4}$ Acadêmico da Faculdade de Medicina da Universidade Federal da Bahia - UFBA.

Professor adjunto-doutor e Livre Docente do Departamento de Medicina, da Faculdade de Medicina da Universidade Federal da Bahia - UFBA.

Endereço para correspondência: Renaldo Duarte Moreno. Rua Floriano Peixoto, 335 - Rio Branco (AC) CEP 69908-030 - E-mail: renaldomoreno@uol.com.br

Recebido para análise em 07.10.2002

Versão revisada recebida em 13.06.2003

Aprovação em 01.07.2003

Nota Editorial: Pela análise deste trabalho e por sua anuência na divulgação desta nota, agradecemos aos Drs. João Orlando Ribeiro Gonçalves e Paulo Ricardo de Oliveira.

\section{RESUMO}

Objetivo: Determinar a prevalência das alterações oculares em pacientes de hanseníase ativa ou inativa, acompanhados em serviço de referência. Métodos: O estudo transversal constou de exame ocular e levantamento de dados demográficos e clínico-epidemiológicos, em serviço de referência da cidade de Rio Branco (Acre), no período de outubro de 2001 a abril de 2002. Resultados: Foram examinados 254 pacientes, com média de idade de 41,9 anos; $70,1 \%$ do sexo masculino e $29,9 \%$ do feminino; $73,6 \%$ residentes da área urbana e 76,8\% de formas multi e 23,2\% de paucibacilares. Anormalidades de anexos oculares foram descritas em 49,6\% ( $n=126)$ dos casos e do globo ocular em $39,4 \%(n=100)$ dos casos, sendo as mais freqüentes: hipoestesia corneana, catarata, madarose, manchas hipercrômicas, hipolacrimejamento e ceratite ponteada. As lesões oculares (74,4\%) tiveram freqüências desiguais $(\rho<0,0001)$, predominando entre aqueles com mais de 40 anos de idade (48,4\% versus $26,0 \%$ nos de 40 anos ou menos), nos casos multibacilares (76,8\% versus $23,2 \%$ ), e nas pessoas com mais de cinco anos de duração da hanseníase $(\rho<0,001)$. As alterações de globo ocular foram mais observadas em pacientes com tratamento específico concluído $(\rho<0,05)$. Conclusões: A freqüência da morbidade ocular na hanseníase, em quase três quartos $(74,4 \%)$ dos casos, foi semelhante à descrita na literatura para pacientes ambulatoriais de serviço de referência, e foi mais prevalente nas formas multibacilares da doença e em pacientes acima de 40 anos de idade.

Descritores: Hanseníase/complicações; Infecções oculares bacterianas/etiologia; Cegueira/etiologia; Hanseníase/epidemiologia; Brasil; Estudos transversais

\section{INTRODUÇÃO}

A hanseníase, ou mal de Hansen (MH), é uma doença crônica, de acometimento sistêmico, causada por bacilo álcool-ácido resistente, o Mycobacterium leprae ${ }^{(1)}$.

Em 1985, a hanseníase foi considerada endêmica em 122 países ${ }^{(2)}$ e, ao final do ano 2000, o Brasil e outros 14 países ainda permaneciam com prevalência superior a um caso por 10.000 habitantes $^{(3)}$. Atualmente, o Brasil ocupa a segunda posição no mundo e a primeira nas Américas, com o diagnóstico de 41.070 casos em $2000^{(2)}$, o que corresponde a mais de $80 \%$ dos casos notificados no continente americano e cerca de $13 \%$ do número global de doentes ${ }^{(2)}$, sendo o coeficiente de prevalência de 4,3 casos por 
756 Alterações oculares na hanseníase, observadas em pacientes ambulatoriais do serviço de referência da cidade de Rio Branco, Acre - Brasil

10.000 habitantes $^{(2)}$. No Estado do Acre, o coeficiente de prevalência em 2001 foi de 5,6 casos por 10.000 habitantes, com 403 casos novos registrados ${ }^{(4)}$.

A hanseníase pode evoluir com apresentações clínicas bastante variadas, incluindo as que lesam o aparelho ocular, tanto pela invasão direta do bacilo quanto por alterações secundárias às inflamações das reações hansênicas ${ }^{(5)}$.

As manifestações oculares mais freqüentes em casos de hanseníase são: hipoestesia corneana, madarose, lagoftamo, catarata, uveíte e opacidades corneanas ${ }^{(6-7)}$. Em conseqüência, a Organização Mundial de Saúde (OMS) estima que o mal de Hansen seja a causa de cegueira em 50.000 a 100.000 pessoas em todo o mundo ${ }^{(8)}$.

Apesar das complicações oculares ocorrerem cerca de 5 a 10 anos após o início da hanseníase ${ }^{(1)}$, cada vez mais são descritas manifestações oculares em pacientes recém-diagnosticados $^{(9-13)}$. Na Amazônia, são escassas as publicações sobre a morbidade ocular na hanseníase ${ }^{(14)}$, daí o objetivo do presente estudo: descrever as alterações oculares em pessoas afetadas pela hanseníase dentro ou fora de atividade da doença, acompanhadas em serviço ambulatorial de referência da cidade de Rio Branco, Acre, durante seis meses.

\section{MÉTODOS}

Este estudo transversal foi realizado no Serviço de Dermatologia Sanitária (SDS-AC), da Secretaria de Saúde do Estado do Acre, localizado na cidade de Rio Branco, no período de outubro de 2001 a abril de 2002. No estudo foram incluídos os pacientes em tratamento específico ou aqueles com tratamento concluído e que procuraram o ambulatório por outros motivos. Como no SDS-AC não havia atendimento regular de Oftalmologia, os motivos das consultas eram de várias naturezas, inclusive sociais.

Os exames oculares foram realizados no SDS-AC, sempre pelo mesmo examinador (RDM) e que desconhecia as informações quanto à forma clínica da doença. O exame ocular constou de: 1) ectoscopia; 2) exame sumário da motilidade ocular; 3) medida da acuidade visual usando optotipos de Snellen; 4) refratometria objetiva e subjetiva com emprego de auto-refrator Topcon ${ }^{\circledR}$, refrator tipo Greens e armação de prova; e sob cicloplegia conforme indicação; 5) biomicroscopia do segmento anterior utilizando lâmpada de fenda modelo Haag-Streit; 6) teste de sensibilidade corneana através do estesiômetro de Cochet \& Bonnet ${ }^{\circledR}$, considerada diminuída se necessário um comprimento $\leq 35 \mathrm{~mm}$ da haste de "nylon" para desencadear o reflexo de piscar; 7) avaliação da secreção lacrimal pelo teste de Schirmer, considerada normal se $\leq 10 \mathrm{~mm}$ de umedecimento do papel de filtro, após 5 minutos de teste, sem anestesia; 8) tonometria ocular de aplanação através de instrumento HaagStreit ${ }^{\circledR}$, considerada normal se entre 10 e $18 \mathrm{mmHg}$; 9) oftalmoscopia direta e indireta sob midríase, com emprego de oftalmoscópios Neitz ${ }^{\circledR}$ e Keeler ${ }^{\circledR}$.

Os pacientes selecionados (ou o responsável legal, se criança), após a concordância em participar do estudo, assinaram termo de consentimento livre e esclarecido aprovado pelo Comitê de Ética em Pesquisa da Fundação Hospital do Estado do Acre (FUNDHACRE, Rio Branco - Acre).

Por ocasião do exame ocular, eram também anotados em ficha-padrão: dados de identificação (nome e registro) do paciente no SDS-AC; data de nascimento; sexo; grupo racial; cidade e local (urbana ou rural) de procedência. Posteriormente, dois examinadores independentes reviram os prontuários e, depois, havendo alguma discordância, o dado clínico era revisto em conjunto por ambos os examinadores. Nessa revisão, além da forma clínica da hanseníase segundo os critérios clínico-bacteriológicos ${ }^{(15)}$, foram registrados: 1) idade de início da doença; 2) duração da doença (anos); 3) tipo de tratamento: PQT (poliquimioterapia: dapsona, rifampicina e clofazemina); Dnds (dapsona isolada); ou ROM (esquema de dose única de rifampicina, ofloxacina e minociclina) ou outro esquema terapêutico; 3) fase atual do tratamento específico, quando do exame ocular (grupo A: concluído; ou do Grupo B: em tratamento); 4) se houve ou não recidiva da hanseníase; 5) resultado do teste de Mitsuda, na época do diagnóstico de hanseníase; 6) a pesquisa de bacilo álcool-ácido-resistente, BAAR (baciloscopia), na época do diagnóstico de hanseníase; e 7) resultado de exame histopatológico, também na época do diagnóstico de hanseníase.

As formas clínicas foram caracterizadas como multibacilares (forma clínica virchowiana e a dimorfa) ou paucibacilares (tuberculóide e a indeterminada), segundo critérios do Ministério da Saúde ${ }^{(16)}$, classificadas respectivamente como do Grupo I ou II.

Quando da revisão do prontuário, foram excluídos os casos sem a caracterização da forma clínica da hanseníase ou sem critérios diagnósticos definidos, bem como os pacientes procedentes de hospital-colônia (da cidade de Rio Branco ou Cruzeiro do Sul).

Todos os casos com anormalidades oculares foram referenciados para tratamento clínico, cirúrgico e/ou fisioterápico no Serviço de Oftalmologia da Fundação Hospital Estadual do Acre (FUNDHACRE).

Os resultados foram registrados na planilha Excel ${ }^{\circledR}$ e analisados pelo programa SPSS ${ }^{\circledR}$ (versão 9.0). Na análise da variável dependente (ou resposta), ou seja, a alteração ocular, foram realizadas comparações com as variáveis demográficas e clínico-laboratoriais, qualitativas ou quantitativas, conforme a indicação estatística. Deste modo, as alterações oculares foram divididas em dois grupos, aquelas dos anexos (no total de 11) e as do globo ocular (no total de 13). Se presente a alteração, de anexo ou globo, o paciente recebia o escore $1 \mathrm{e}$ dessa forma cada pessoa poderia ter, respectivamente, de 0 a 11 pontos e de 0 a 13 pontos. Nessas análises, foi aplicado o teste não-paramétrico de Mann-Whitney para estudar a distribuição dos escores, de cada grupo de alterações (anexos e globo), de acordo com: 1) sexo; 2) idade do paciente; 3) idade de início da doença; 4) duração da doença; 5) história de recidiva, sim ou não; 6) resultado da baciloscopia, se positiva 
ou negativa; 7) as formas paucibacilares versus multibacilares; e, 8) fase do tratamento específico.

Após as análises univariadas, aquelas variáveis com associação $(\rho \leq 0,25)$ à variável-resposta foram incluídas no teste multifatorial, de regressão logística. Nessas análises estatísticas, a probabilidade $(\rho)$ do erro tipo I $(\alpha)$ foi considerada significante se $\leq 5 \%(\rho \leq 0,05)$ ou altamente significante quando $\leq 1 \%(\rho \leq 0,01)$.

\section{RESULTADOS}

Dos 269 pacientes examinados, 15 (5,6\%) foram posteriormente excluídos das análises, porque não preencheram os critérios de inclusão. Entre os pacientes incluídos (n=254), as freqüências de anormalidades oculares foram de 49,6\% $(n=126)$ em anexos e 39,4\% $(n=100)$ de bulbo ocular. Das pessoas examinadas, 65 (25,6\%) não apresentavam morbidade ocular associada à hanseníase.

Entre os 254 pacientes de hanseníase (Tabela 1), a proporção de pessoas do sexo masculino foi de $70,1 \%$ e $29,9 \%$ do feminino. A idade variou de 5,6 a 85,7 anos, com média de 41,9 $( \pm 18,1)$, sendo a faixa etária de maior ocorrência de 41 a 50 anos, com $56(22,0 \%)$ pacientes. Quanto ao grupo racial, predominou $(65,0 \%)$ o das pessoas classificadas como mulatas. A maioria (60,0\%) dos pacientes procedia do município de Rio Branco e, no conjunto, 73,6\% de áreas urbanas. Em concordância com a distribuição etária da amostra, quase a metade (49,6\%) era de pessoas casadas.

Também na tabela 1, as características demográficas foram analisadas quanto às formas clínicas da hanseníase, multibacilar $(76,8 \%)$ versus paucibacilar $(23,2 \%)$, correspondendo,

Tabela 1. Características demográficas de 254 pacientes de hanseníase, conforme a forma clínica associada a baciloscopia (paucibacilar ou multibacilar). Rio Branco, Acre, 2002

\begin{tabular}{|c|c|c|c|c|}
\hline \multirow[b]{2}{*}{ Características } & \multicolumn{3}{|c|}{ Clínica e baciloscopia } & \multirow[b]{2}{*}{$\rho$} \\
\hline & $\begin{array}{c}\text { Total } \\
(n=254)\end{array}$ & $\begin{array}{l}\text { Multibacilar } \\
\quad(n=195)\end{array}$ & $\begin{array}{l}\text { Paucibacilar } \\
(n=59)\end{array}$ & \\
\hline Idade (anos) & 41,9 & 42,7 & 39,6 & $>0,26^{\mathrm{a}}$ \\
\hline Média (DP) & $(18,1)$ & $(18,2)$ & $(17,7)$ & \\
\hline Limites & $5,6|-| 85,7$ & $5,6|-| 84,1$ & $11,4|-| 85,7$ & \\
\hline Faixa etáriab (anos), n (\%) & & & & $>0,50^{(\mathrm{c}) d}$ \\
\hline$\leq 10^{d}$ & $2(0,8)$ & $2(1,0)$ & $0 \quad(0,0)$ & \\
\hline $11|-| 20^{d}$ & $41(16,1)$ & $29(14,9)$ & $12(20,3)$ & \\
\hline $21|-| 30$ & $41(16,1)$ & $30(15,4)$ & $11(18,6)$ & \\
\hline $31|-| 40$ & $31 \quad(12,2)$ & $25(12,8)$ & $6(10,2)$ & \\
\hline $41|-| 50$ & $56(22,0)$ & $43(22,1)$ & $13(22,0)$ & \\
\hline $51|-| 60$ & $38(15,0)$ & $30(15,4)$ & $8(13,6)$ & \\
\hline $61|-| 70^{d}$ & $30(11,8)$ & $22(11,3)$ & $8(13,6)$ & \\
\hline$>70^{d}$ & $15(5,9)$ & $14(7,2)$ & $1(1,7)$ & \\
\hline Sexo, n (\%) & & & & $<0,0008^{c}$ \\
\hline Masculino & $178(70,1)$ & $147(75,4)$ & $31(52,5)$ & \\
\hline Feminino & $76(29,9)$ & $48(24,6)$ & $28(47,5)$ & \\
\hline Grupo racial, n (\%) & & & & $>0,79^{c}$ \\
\hline Branco & $25 \quad(9,9)$ & $19(9,7)$ & $6(10,2)$ & \\
\hline Mulato & $165(65,0)$ & $125(64,1)$ & $40(67,8)$ & \\
\hline Negro $^{e}$ & $4(1,6)$ & $3(1,5)$ & $1(1,7)$ & \\
\hline Mestiço de índio & $59(23,4)$ & $47 \quad(24,1)$ & $12(20,3)$ & \\
\hline Indígena ${ }^{f}$ & $1(0,4)$ & $1(0,5)$ & $0(0,0)$ & \\
\hline Procedência, n (\%) & & & & $>0,17^{c}$ \\
\hline Rio Branco & $150(60,0)$ & $109(55,9)$ & $41 \quad(69,5)$ & \\
\hline Interior & $54(21,0)$ & $53(27,2)$ & $11(18,6)$ & \\
\hline Outros Estados & $50(19,0)$ & $33(16,9)$ & $7(11,9)$ & \\
\hline Estado civil, n (\%) & & & & $>0,51^{c}$ \\
\hline Solteiro & $92(36,2)$ & $74 \quad(37,9)$ & $18(30,5)$ & \\
\hline Casado & $126(49,6)$ & $93(47,7)$ & $33(55,9)$ & \\
\hline Outros & $36(14,2)$ & $28(14,3)$ & $8(13,6)$ & \\
\hline Residência, n (\%) & & & & $<0,01^{c}$ \\
\hline Urbana & $187(73,6)$ & $136(69,7)$ & $51(86,4)$ & \\
\hline Rural & $67(26,4)$ & $59(30,3)$ & $8(13,6)$ & \\
\hline
\end{tabular}


758 Alterações oculares na hanseníase, observadas em pacientes ambulatoriais do serviço de referência da cidade de Rio Branco, Acre - Brasil

respectivamente, a 195 e 59 pacientes. A distribuição dessas formas foi estatisticamente semelhante quanto à idade $(\rho>0,26)$, faixa etária $(\rho>0,50)$, grupo racial $(\rho>0,79)$, procedência $(\rho>0,17)$ e estado civil $(\rho>0,51)$. A freqüência de pessoas do sexo masculino entre os multibacilares $(75,4 \%$ versus $24,6 \%)$ foi três vezes maior e aproximadamente de 1:1 (52,5\% versus $47,5 \%$ ) no grupo paucibacilar, sendo essa diferença altamente significante $(\rho<0,0008)$. Apesar de ambos os grupos serem mais freqüentes entre as pessoas das áreas urbanas (I: 69,7\% e II: 86,4\%), aquelas de áreas rurais foram aproximadamente duas (30,3\%) e seis $(13,6 \%)$ vezes menos freqüentes nos grupos I e II, respectivamente, sendo essa diferença altamente significante $(\rho<0,01)$.

Entre os 254 casos estudados, as formas clínicas da hanseníase tiveram a seguinte distribuição: $28,4 \%(n=72)$ virchowiana (V); 48,4\% (n=123) da dimorfa (D); 20,5\% (n=52) tuberculóide (T) e somente $2,8 \%(n=7)$ com a forma clínica indeterminada (I). Nas análises estatísticas subseqüentes, por causa da baixa freqüência, algumas vezes os casos com a forma I foram somados aos da forma T, e, em outras vezes, excluídos. Na tabela 2, essas formas clínicas foram estudadas segundo alguns indicadores clínico-epidemiológicos. A média de idade de início da doença foi de 30,3 $( \pm 15,9)$ anos, não diferindo $(\rho>0,84)$ entre as formas clínicas. $\mathrm{O}$ tempo de duração da hanseníase foi em média de 10,8 $( \pm 14,3)$ anos, sendo o das formas $\mathrm{V}$ de maior duração (18,6 $\pm 18,4$ anos) e o das formas I as de menor $(5,2$ $\pm 8,0$ anos), mas sem significância estatística $(\rho>0,78)$.

Também como mostra a tabela 2 , houve predomínio $(\rho<0,02)$ de pessoas com tratamento específico concluído $(57,1 \%)$ ou, mais especificamente, entre aquelas das formas $\mathrm{V}$ $(69,4 \%)$ e $\mathrm{T}(61,5 \%)$. A freqüência de recidiva da doença foi de $14,6 \%$ (35/240) e, excluindo os da forma I (2/6), a distribuição foi semelhante $(\rho>0,48)$ entre os casos das 3 outras formas clínicas. Na população estudada, somente 30 pacientes foram submetidos ao teste de Mitsuda, e a freqüência de reatores foi semelhante $(\rho>0,43)$ no grupo multibacilar $(V+D)$ versus os da forma $\mathrm{T}$ (paucibacilar). Um número menor ainda de pacientes $(n=10)$ tinha exame histopatológico, todos com resultado compatível com a forma clínica registrada em prontuário.

Em 16 (6,3\%) pacientes, ainda na tabela 2, a pesquisa de BAAR (baciloscopia) não foi encontrada em prontuário. Excluídos os 6 casos com a forma clínica Indeterminada (todos BAAR-negativos), a freqüência de baciloscopia foi marcadamente $\left(\rho<10^{-9}\right)$ positiva nos casos virchowianos $(80,9 \%$ ou

\begin{tabular}{|c|c|c|c|c|c|c|}
\hline \multirow[b]{2}{*}{ Dado clínico } & \multicolumn{5}{|c|}{ Forma clínica } & \multirow[b]{2}{*}{$\rho$} \\
\hline & Total & $\mathbf{V}^{(\mathbf{a})}$ & $D^{(b)}$ & $\mathbf{T}^{(\mathrm{c})}$ & $I^{(d)}$ & \\
\hline Idade do início da doença & 30,3 & 31,1 & 30,3 & 28,7 & 32,4 & $>0,84^{\dagger}$ \\
\hline Média (DP) ${ }^{e}$ & $(15,9)$ & $(17,0)$ & $(15,8)$ & $(15,1)$ & $(11,0)$ & \\
\hline Limites & $4|-| 72$ & $6|-| 63$ & $5|-| 70$ & $4|-| 72$ & $13|-| 44$ & \\
\hline Duração da doença & 10,8 & 18,6 & 6,9 & 10,3 & 5,2 & $>0,78^{9}$ \\
\hline Média $(D P)^{e}$ & $(14,3)$ & $(18,4)$ & $(10,0)$ & $(13,1)$ & $(8,0)$ & \\
\hline Limites & $01|-| 65$ & $1,3|-| 65$ & $0,2|-| 47$ & $0,1|-| 58$ & $0,2|-| 20$ & \\
\hline \multicolumn{2}{|l|}{ Tipo de tratamento, n (\%) } & & & & & $<0,0005^{(h) i}$ \\
\hline$P Q T$ & $215(84,6)$ & $53(73,6)$ & $115(93,5)$ & $41(78,8)$ & $6(85,7)$ & \\
\hline Dnds $^{h}$ & $26(10,2)$ & $15(20,8)$ & $2(1,6)$ & $8(15,4)$ & $1(14,3)$ & \\
\hline $\mathrm{ROM}^{\mathrm{h}}$ & $2(0,8)$ & $0(0,0)$ & $0(0,0)$ & $2(3,8)$ & $0(0,0)$ & \\
\hline Outros ${ }^{h}$ & $1(0,4)$ & $1(1,4)$ & $0(0,0)$ & $0(0,0)$ & $0(0,0)$ & \\
\hline Ignorado $^{h}$ & $10(3,9)$ & $3(4,2)$ & $6(4,9)$ & $1(1,9)$ & $0(0,0)$ & \\
\hline \multicolumn{2}{|l|}{ Fase do tratamento, $\mathrm{n}(\%)$} & & & & & $<0,02^{(i) j}$ \\
\hline Concluído & $145(57,1)$ & $50(69,4)$ & $60(48,8)$ & $32(61,5)$ & $3(42,9)$ & \\
\hline Em andamento & $109(42,9)$ & $22(30,6)$ & $63(51,2)$ & $20(38,5)$ & $4(57,1)$ & \\
\hline \multicolumn{2}{|l|}{ Recidiva, n (\%) } & & & & & $>0,48^{(i) k}$ \\
\hline Sim & $35(13,8)$ & $12(16,7)$ & $16(13,0)$ & $5(9,6)$ & $2(28,6)$ & \\
\hline Não & $205(80,7)$ & $55(76,4)$ & $102(82,9)$ & $44(84,6)$ & $4(57,1)$ & \\
\hline Sem informação & $14(5,5)$ & $5(6,9)$ & $5(4,1)$ & $3(5,8)$ & $1(14,3)$ & \\
\hline \multicolumn{2}{|l|}{ Teste de Mitsuda, n (\%) } & & & & & $>0,43^{(1) !}$ \\
\hline Reator & $21(8,3)$ & $1(1,4)$ & $7(5,7)$ & $13(25,0)$ & $0(0,0)^{m}$ & \\
\hline Não-reator & $9(3,5)$ & $3(4,2)$ & $1(0,8)$ & $4(7,7)$ & $1(14,3)^{m}$ & \\
\hline Não realizadom & $224(88,2)$ & $68(94,4)$ & $115(93,5)$ & $35(67,3)$ & $6(85,7)^{\mathrm{m}}$ & \\
\hline \multicolumn{2}{|l|}{ Baciloscopia, n (\%) } & & & & & $<10^{-9(i) n}$ \\
\hline Positiva & $99(39,0)$ & $51(70,8)$ & $48(39,0)$ & $0 \quad(0,0)$ & $0(0,0)$ & \\
\hline Negativa & $139(54,7)$ & $12(16,7)$ & $69(56,1)$ & $52(100,0)$ & $6(85,7)$ & \\
\hline Não realizada & $16(6,3)$ & $9(12,5)$ & $6(4,9)$ & $0 \quad(0,0)$ & $1(14,3)$ & \\
\hline \multicolumn{7}{|c|}{$\begin{array}{l}\text { (a) virchowiana; (b) dimorfa; (c) tuberculóide; (d) indeterminada; (e) em anos; (f) teste ANOVA; (g) Teste de Kruskal-Wallis; (h) exclusão dos casos da forma I: PQT } \\
\text { versus demais esquemas; (i) qui-quadrado; (j) excluídos os casos da forma I; (k) excluídos aqueles sem informação e os casos da forma I; (I) teste exato de Fisher; } \\
\text { (m) excluídos os casos sem o teste e os da forma I, comparando os da forma T versus (V + D); (n) excluídos os casos sem baciloscopia e os da forma I }\end{array}$} \\
\hline
\end{tabular}


cidade de Rio Branco, Acre - Brasil

51/63), sendo entre os dimórficos de 41,0\% (48/117) e de $0 \%$ (0/52) nos da forma $T$.

A medida da acuidade visual (Tabela 3), de 227 (89,7\%) pacientes, foi considerada boa ou normal (20/20 a 20/40) em pelo menos um dos olhos ("olho de melhor visão"). A deficiência visual moderada (20/50 a 20/70), grave (20/80 a 20/200) e a cegueira (visão menor que 20/200) tiveram freqüências, respectivamente, de $4,3 \%(n=11), 4,0 \%(n=10)$ e $2,0 \%(n=5)$ pacientes. Devido ao pequeno número de casos $(n=26)$ com déficit visual (distribuído nos três graus de redução da acuidade visual), nas análises seguintes, aquela classificada como "boa ou normal" (no olho de melhor visão) foi comparada às demais. Sendo assim, a freqüência de visão normal foi significativamente menor $(\rho<0,003)$ nas pessoas maiores de 40 anos de idade (84,0\%), do que naquelas com 40 anos ou menos de idade (96,4\%). Além dessa associação com a idade, o grupo de pessoas com tratamento concluído tinha, significativamente $(\rho<0,0007)$, menor freqüência $(84,0 \%)$ de visão normal, quando comparadas às pessoas com tratamento em curso $(97,2 \%)$. No entanto, não houve diferença estatística $(\rho>0,13)$ quanto à acuidade visual (boa versus outras) entre os casos com as formas multibacilares (grupo I: V + D) e paucibacilares (grupo II: T + I), apesar da menor freqüência $(88,1 \%)$ de visão normal nas pessoas do grupo I, comparadas aos do II (94,9\%).

Nos anexos oculares, as alterações oculares mais freqüentes observadas foram a madarose de cílios (20,5\%) e de supercílios (16,1\%), manchas palpebrais hipercrômicas (14,2\%), hipolacrimejamento (14,2\%), hipotrofia palpebral (9,4\%), eritema nodoso palpebral (6,7\%) e lagoftalmo (6,7\%). Entre as alterações oculares propriamente ditas, ou de bulbo ocular, as mais freqüentes foram: hipoestesia corneana (27,6\%), catarata (20,9\%), manchas conjuntivais $(15,7 \%)$, ceratite ponteada (11,4\%), hipotrofia da íris (9,4\%) e leucomas (6,7\%).

Na tabela 4, as alterações de anexos oculares foram distribuídas entre as formas clínicas (multibacilares e paucibacilares), algumas delas significativamente mais freqüentes nas

\begin{tabular}{|c|c|c|c|c|c|c|c|c|c|}
\hline \multirow[b]{3}{*}{ Pacientes } & \multicolumn{9}{|c|}{ Acuidade visual, $n(\%)$} \\
\hline & \multirow[t]{2}{*}{ Boab $^{b}$} & \multicolumn{2}{|c|}{ Dmod $^{c}$} & Dgrad $^{d}$ & \multicolumn{2}{|c|}{ Ceg. ${ }^{e}$} & \multicolumn{2}{|c|}{ Total } & \multirow[b]{2}{*}{$(100,0) \rho$} \\
\hline & & $(89,7)$ & 11 & $(4,3)$ & $(4,0)$ & 5 & $(2,0)$ & 253 & \\
\hline \multicolumn{10}{|l|}{ Idade (anos) } \\
\hline$\leq 40$ & $106(96,4)$ & 0 & $(0,0)$ & $2(1,8)$ & 2 & $(1,8)$ & 110 & & \multirow[t]{2}{*}{$<0,003^{f}$} \\
\hline$>40$ & $121(84,6)$ & 11 & $(7,7)$ & $8(5,6)$ & 3 & $(2,1)$ & 143 & & \\
\hline \multicolumn{10}{|l|}{ Forma clínica } \\
\hline Multibacilar & $171(88,1)$ & 8 & $(4,1)$ & $10(5,2)$ & 5 & $(2,6)$ & 193 & & \multirow[t]{2}{*}{$>0,13^{f}$} \\
\hline Paucibacilar & $56(94,9)$ & 3 & $(5,1)$ & $0(0,0)$ & 0 & $(0,0)$ & 59 & & \\
\hline \multicolumn{10}{|l|}{ Fase do tratamento } \\
\hline Concluído & $121(84,0)$ & 10 & $(6,9)$ & $8(5,6)$ & 5 & $(3,5)$ & 144 & & \multirow[t]{2}{*}{$<0,0007^{\dagger}$} \\
\hline Em tratamento & $106(97,2)$ & 1 & $(0,9)$ & $2(1,8)$ & 0 & $(0,0)$ & 109 & & \\
\hline
\end{tabular}

Tabela 4. Freqüência das alterações de anexos oculares em 254 pacientes de hanseníase, segundo a característica clínica (multi e paucibacilar) da doença. Rio Branco, Acre, 2002

\begin{tabular}{|c|c|c|c|c|}
\hline \multirow[b]{2}{*}{ Alterações } & \multirow[b]{2}{*}{$\begin{array}{c}\text { Total } \\
(n=254)\end{array}$} & \multicolumn{2}{|c|}{ Forma clínica, n (\%) } & \multirow{3}{*}{$\underset{<0,003^{b}}{\rho}$} \\
\hline & & $\begin{array}{c}\text { Multibacilar } \\
(n=195)\end{array}$ & $\begin{array}{c}\text { Paucibacilar } \\
(n=59)\end{array}$ & \\
\hline Madarose de cílios & $52(20,5)$ & $48 \quad(24,6)$ & $4(6,8)$ & \\
\hline Madarose de supercílios & $41(16,1)$ & $37(19,0)$ & $4(6,8)$ & $<0,03^{\mathrm{b}}$ \\
\hline Manchas palpebrais & $36(14,2)$ & $34 \quad(17,4)$ & $2(3,4)$ & $<0,0007^{b}$ \\
\hline Hipolacrimejamento ${ }^{a}$ & $30(14,2)$ & $19 \quad(11,9)$ & $11(21,6)$ & $>0,06^{b}$ \\
\hline Hipotrofia palpebral & $24(9,4)$ & $22 \quad(11,3)$ & $2(3,4)$ & $>0,06^{b}$ \\
\hline Eritema nodoso palpebral & $17(6,7)$ & $16 \quad(8,2)$ & $1(1,7)$ & $>0,14^{c}$ \\
\hline Lagoftalmo & $17(6,7)$ & $14(7,2)$ & $3(5,1)$ & $>0,78^{c}$ \\
\hline Triquíase & $9(3,5)$ & $8 \quad(4,1)$ & $1(1,7)$ & $>0,62^{c}$ \\
\hline Epífora & $5 \quad(2,0)$ & $4 \quad(2,1)$ & $1(1,7)$ & $>0,67^{d}$ \\
\hline Ectrópio & $4(1,6)$ & $3(1,5)$ & $1(1,7)$ & $>0,65^{d}$ \\
\hline Dacriocistite & $3(1,2)$ & $3(1,5)$ & $0(0,0)$ & $>0,45^{d}$ \\
\hline
\end{tabular}


760 Alterações oculares na hanseníase, observadas em pacientes ambulatoriais do serviço de referência da cidade de Rio Branco, Acre - Brasil

multibacilares (madaroses e manchas palpebrais) ou próximo ao limite de significância $(\rho>0,06)$ : hipolacrimejamento e hipotrofia palpebral. As demais alterações tiveram freqüências semelhantes $(\rho>0,14)$ entre as formas multi e paucibacilares. Quando essas mesmas alterações foram distribuídas segundo a fase de tratamento específico (concluído versus em andamento), predominaram significativamente no grupo "concluído" as seguintes alterações (Tabela 5): madaroses; hipotrofia palpebral e triquíase; e epífora (no limite de significância, $\rho>0,06)$. Já as manchas palpebrais $(\rho<0,0001)$ e o eritema nodoso palpebral $(\rho<0,02)$ predominaram no grupo em tratamento.

Análises semelhantes foram realizadas sobre as alterações do globo ocular (Tabelas 6 e 7). A tabela 6 mostra que houve predominância significativa no grupo multibacilar de manchas conjuntivais $(\rho<0,04)$, leucomas $(\rho<0,02)$ e estando no limite de significância $(\rho>0,06)$ a hipotrofia de íris. Nenhuma dessas alterações foi mais freqüente no grupo paucibacilar, e as de- mais alterações descritas na tabela 6 foram semelhantes $(\rho>0,08)$ nos dois grupos. Quanto à fase de tratamento (Tabela $7)$, os tipos de alterações significativamente mais comuns no grupo com tratamento específico concluído foram: catarata $(\rho<0,003)$, hipotrofia de íris $(\rho<0,002)$ e pupila miótica $(\rho<0,03)$, enquanto que as manchas conjuntivais prevaleceram $(\rho<0,0007)$ entre aqueles ainda em tratamento.

Em seguimento, aplicou-se o teste estatístico de MannWhitney, que revelou pacientes com alterações de anexos oculares cujos escores variaram de 0 a 6 (escala de 0 a 11) com mediana de 1 ponto, sendo no grupo das alterações de globo ocular com escores variando de 0 a 10 (escala de 0 a 13) e mediana também de 1 ponto. Observa-se na tabela 8 que a ocorrência de morbidade ocular, tanto de anexo quanto de globo, foram significativamente mais freqüentes nos pacientes acima de 40 anos de idade (próximo ao limite da significância, quando a doença teve início após os 30 anos de idade), do

\begin{tabular}{|c|c|c|c|c|}
\hline \multirow[b]{2}{*}{ Alterações } & \multirow[b]{2}{*}{$\begin{array}{c}\text { Total } \\
(n=254)\end{array}$} & \multicolumn{2}{|c|}{ Fase do tratamento, $\mathrm{n}(\%)$} & \multirow{3}{*}{$\begin{array}{c}\rho \\
<0,0004^{b}\end{array}$} \\
\hline & & $\begin{array}{c}\text { Concluído } \\
(n=145)\end{array}$ & $\begin{array}{c}\text { Em andamento } \\
(n=109)\end{array}$ & \\
\hline Madarose de cílios & $52(20,5)$ & $41(28,3)$ & $11(10,1)$ & \\
\hline Madarose de supercílios & $41(16,1)$ & $36(24,8)$ & $5(4,6)$ & $<0,0002^{b}$ \\
\hline Manchas palpebrais & $36(14,2)$ & $10(6,9)$ & $26(23,9)$ & $<0,0001^{b}$ \\
\hline Hipolacrimejamento ${ }^{a}$ & $30(14,2)$ & $17(11,7)$ & $13(11,9)$ & $>0,95^{b}$ \\
\hline Hipotrofia palpebral & $24 \quad(9,4)$ & $21(14,5)$ & $3(2,8)$ & $<0,002^{b}$ \\
\hline Eritema nodoso palpebral & $17(6,7)$ & $5(3,4)$ & $12(11,0)$ & $<0,02^{b}$ \\
\hline Lagoftalmo & $17(6,7)$ & $12(8,3)$ & $5(4,6)$ & $>0,23^{b}$ \\
\hline Triquíase & $9(3,5)$ & $8(5,5)$ & $1(1,9)$ & $<0,05^{c}$ \\
\hline Epífora & $5 \quad(2,0)$ & $5(3,4)$ & $0(0,0)$ & $>0,06^{c}$ \\
\hline Ectrópio & $4(1,6)$ & $4(2,8)$ & $0(0,0)$ & $>0,10^{c}$ \\
\hline Dacriocistite & $3(1,2)$ & $2(1,4)$ & $1(0,9)$ & $>0,59^{c}$ \\
\hline
\end{tabular}

\begin{tabular}{|c|c|c|c|c|}
\hline \multirow[b]{2}{*}{ Alterações do globo } & \multirow[b]{2}{*}{$\begin{array}{c}\text { Total } \\
(n=254)\end{array}$} & \multicolumn{2}{|c|}{ Forma clínica, n (\%) } & \multirow[b]{2}{*}{$\rho$} \\
\hline & & $\begin{array}{c}\text { Multibacilar } \\
(n=195)\end{array}$ & $\begin{array}{l}\text { Paucibacilar } \\
(n=59)\end{array}$ & \\
\hline Hipoestesia corneana & $70(27,6)$ & $59(30,3)$ & $11(18,6)$ & $>0,08^{b}$ \\
\hline Catarata & $53(20,9)$ & $44(22,6)$ & $9(15,3)$ & $>0,22^{b}$ \\
\hline Manchas conjuntivais & $40(15,7)$ & $36(18,5)$ & $4(6,8)$ & $<0,04^{\mathrm{b}}$ \\
\hline Ceratite ponteada & $29(11,4)$ & $24(12,3)$ & $5(8,5)$ & $>0,41^{b}$ \\
\hline Hipotrofia de íris & $24(9,4)$ & $22(11,3)$ & $2(3,4)$ & $>0,06^{b}$ \\
\hline Leucomas & $17(6,7)$ & $17(8,7)$ & $0(0,0)$ & $<0,02^{b}$ \\
\hline Pupila miótica & $14(5,5)$ & $13(6,7)$ & $1(1,7)$ & $>0,14^{b}$ \\
\hline Glaucoma $^{\mathrm{a}}$ & $12(5,9)$ & $9(5,8)$ & $3(6,5)$ & $>0,84^{c}$ \\
\hline Cicatrizes de coriorretinite & $12(4,7)$ & $9(4,6)$ & $3(5,1)$ & $>0,84^{c}$ \\
\hline Sinéquias iridocristalinianas & $7(2,8)$ & $7(3,6)$ & $0(0,0)$ & $>0,13^{\circ}$ \\
\hline Uveíte & $6(2,4)$ & $5(2,6)$ & $1(1,7)$ & $>0,15^{\mathrm{d}}$ \\
\hline Esclerite/Episclerite & $6(2,4)$ & $5(2,5)$ & $1(1,7)$ & $>0,58^{\circ}$ \\
\hline Úlcera de córnea & $2(0,8)$ & $1(0,5)$ & $1(1,7)$ & $>0,41^{\circ}$ \\
\hline
\end{tabular}


sexo masculino, com duração da hanseníase maior de 5 anos e entre aqueles da forma multibacilar, ficando no limite de significância estatística (anexos: $\rho>0,05$ ) ou significante (globo: $\rho<0,05$ ) nos casos de tratamento específico concluído. Não foi observada associação de lesões oculares com o relato de recidiva de hanseníase (anexos: $\rho>0,21$; oculares: $\rho>0,07$ ).

$\mathrm{Na}$ análise multifatorial, utilizando a regressão logística para avaliar as variáveis independentes, a idade acima dos 40 anos $(\mathrm{OR}=3,95 ; 95 \%$ IC de 2,12 - 7,36; $\rho<0,001)$ e as formas clínicas multibacilares (OR= 2,33; 95\% IC de 1,20 - 4,55; $\rho<0,01)$ foram as mais determinantes da ocorrência da morbidade ocular.

Dos 5 pacientes considerados cegos, 2 (40,0\%) apresentavam catarata isoladamente. Nos demais $(60,0 \%)$, além da catarata, observou-se comprometimento da córnea (opacidades) e/ou da úvea (hipotrofia da íris e pupila miótica).

Em nenhum paciente foram diagnosticadas a atrofia e a hipotensão oculares. À fundoscopia, alguns achados foram

\begin{tabular}{|c|c|c|c|c|}
\hline \multirow[b]{2}{*}{ Alterações do globo } & \multirow[b]{2}{*}{$\begin{array}{c}\text { Total } \\
(n=254)\end{array}$} & \multicolumn{2}{|c|}{ Fase do tratamento $\mathrm{n}(\%)$} & \multirow[b]{2}{*}{$\rho$} \\
\hline & & $\begin{array}{c}\text { Concluído } \\
(n=145)\end{array}$ & $\begin{array}{c}\text { Em andamento } \\
(n=109)\end{array}$ & \\
\hline Hipoestesia corneana & $70(27,6)$ & $46(31,7)$ & $24(22,0)$ & $>0,08^{b}$ \\
\hline Catarata & $53(20,9)$ & $40(27,6)$ & $13(11,9)$ & $<0,003^{b}$ \\
\hline Manchas conjuntivais & $40(15,7)$ & $13(9,0)$ & $27(24,8)$ & $<0,0007^{b}$ \\
\hline Ceratite ponteada & $29(11,4)$ & $16(11,0)$ & $13(11,9)$ & $>0,82^{b}$ \\
\hline Hipotrofia de íris & $24(9,4)$ & $21(14,5)$ & $3(2,8)$ & $<0,002^{b}$ \\
\hline Leucomas & $17(6,7)$ & $11(7,6)$ & $6(5,5)$ & $>0,51^{b}$ \\
\hline Pupila miótica & $14(5,5)$ & $12(8,3)$ & $2(1,8)$ & $<0,03^{b}$ \\
\hline Glaucoma $^{a}$ & $12(5,9)$ & $8(5,5)$ & $4(3,7)$ & $>0,49^{b}$ \\
\hline Cicatrizes de coriorretinite & $12(4,7)$ & $8(5,5)$ & $4(3,7)$ & $>0,49^{b}$ \\
\hline Sinéquias iridocristalinianas & $7(2,8)$ & $6(4,1)$ & $1(0,9)$ & $>0,12^{c}$ \\
\hline Uveíte & $6(2,4)$ & $4(2,8)$ & $2(1,8)$ & $>0,48^{c}$ \\
\hline Esclerite/ Episclerite & $6(2,4)$ & $5(3,5)$ & $1(0,9)$ & $>0,18^{c}$ \\
\hline Úlcera de córnea & $2(0,8)$ & $2(1,4)$ & $0(0,0)$ & $>0,32^{c}$ \\
\hline
\end{tabular}

\begin{tabular}{|c|c|c|c|c|c|c|}
\hline \multirow[b]{2}{*}{ Variáveis } & \multicolumn{3}{|c|}{ Anexos oculares } & \multicolumn{3}{|c|}{ Globo ocular } \\
\hline & Média (DP) & Mediana & $\rho$ & Média (DP) & Mediana & $\rho$ \\
\hline Sexo & & & $<0,03^{a}$ & & & $<0,04^{a}$ \\
\hline Masculino & $1,1(1,3)$ & 1,0 & & $1,3(1,5)$ & 1,0 & \\
\hline Feminino & $0,7(1,0)$ & $<0,1$ & & $0,9(1,2)$ & 1,0 & \\
\hline Idade atual & & & $<0,0001^{a}$ & & & $<0,0001^{a}$ \\
\hline$\leq 40$ & $0,5(0,9)$ & $<0,1$ & & $0,6(1,8)$ & $<0,1$ & \\
\hline$>40$ & $1,3(1,3)$ & 1,0 & & $1,6(1,6)$ & 1,0 & \\
\hline Idade de início da hanseníase & & & $>0,05^{a}$ & & & $>0,05^{a}$ \\
\hline$\leq 30$ & $0,9(1,3)$ & $<0,1$ & & $1,0(1,4)$ & 1,0 & \\
\hline$>30$ & $1,0(1,1)$ & 1,0 & & $1,3(1,4)$ & 1,0 & \\
\hline Duração da hanseníase & & & $<0,000^{a}$ & & & $<0,0001^{a}$ \\
\hline$\leq 5$ & $0,7(1,0)$ & $<0,1$ & & $0,9(1,0)$ & 1,0 & \\
\hline$>5$ & $1,3(1,3)$ & 1,0 & & $1,6(1,8)$ & 1,0 & \\
\hline Recidiva da hanseníase & & & $>0,21^{a}$ & & & $>0,07^{a}$ \\
\hline Sim & $1,1(1,3)$ & 1,0 & & $1,5(1,9)$ & 1,0 & \\
\hline Não & $0,9(1,2)$ & $<0,1$ & & $1,0(1,3)$ & 1,0 & \\
\hline Forma clínica da hanseníase & & & $<0,001^{a}$ & & & $<0,009^{a}$ \\
\hline Multibacilar & $1,1(1,3)$ & 1,0 & & $1,3(1,5)$ & 1,0 & \\
\hline Paucibacilar & $0,5(0,8)$ & $<0,1$ & & $0,7(0,8)$ & $<0,1$ & \\
\hline Fase do tratamento & & & $>0,05^{a}$ & & & $<0,05^{a}$ \\
\hline Concluído & $1,1(1,3)$ & 1,0 & & $1,3(1,6)$ & 1,0 & \\
\hline Em andamento & $0,7(0,9)$ & $<0,1$ & & $0,9(1,1)$ & 1,0 & \\
\hline (a) Teste de Mann-Whitney & & & & & & \\
\hline
\end{tabular}


762 Alterações oculares na hanseníase, observadas em pacientes ambulatoriais do serviço de referência da cidade de Rio Branco, Acre - Brasil

considerados inespecíficos, como retinopatia diabética em um paciente e vasculares, em outros 15 (5,9\%). Os erros de refração ocular foram identificados em $65,4 \%(n=166)$ dos pacientes, adicionando-se a presbiopia. Também freqüente foi o pterígio, presente em $11,8 \%(n=30)$ dos examinados.

\section{DISCUSSÃO}

As alterações oculares que acompanham o mal de Hansen devem ser avaliadas segundo os aspectos demográficos, ambientais e da história natural da doença. Contudo, as dificuldades para dimensionar a extensão das alterações do órgão visual começam na definição da metodologia aplicada nos diferentes estudos. Revisão abrangente da literatura verificou alterações oculares em 15\% a 100\% dos pacientes abrigados em hospitais-colônia ${ }^{(17)}$. Da mesma forma, em casuística semelhante, de hospital-colônia da cidade de Rio Branco (Acre), as alterações oculares estiveram presentes em $100 \%$ dos 63 pacientes abrigados, e as mais freqüentes foram: triquíase, madarose, hipoestesia corneana e lagoftalmo ${ }^{(18)}$.

Assim, a prevalência da morbidade ocular na hanseníase tem ampla variação (6\% a 100\%), de acordo com a procedência da população estudada, o método e as formas clínicas ${ }^{(17,19-20)}$. Neste estudo, com casuística selecionada a partir de unidade de saúde ambulatorial, aproximadamente três quartos (74,4\%) tinham alguma alteração ocular, semelhante à proporção observada no Distrito Federal $(70 \%)^{(21)}$ e mais do dobro do que foi encontrado no Estado do Amazonas (32\%) ${ }^{(14)}$.

Entre os dados demográficos das pessoas afetadas pela hanseníase, destaca-se o maior envolvimento das do sexo masculino na proporção de 3:1, concordante com a literatura mundial $^{(22-24)}$, brasileira ${ }^{(10,19,21)}$ e regional $^{(14)}$. Também quanto à média de idade (41,9 \18,1 anos) da população estudada, há semelhança com as descritas em outras publicações ${ }^{(14,22,24)}$. Coincidentemente, o padrão da doença tem se mantido no Estado do Acre, como se pode observar no relatório divulgado pelo jornal "Folha do Acre", de 11 de agosto de 1929: “... abrigavam-se 28 doentes no estabelecimento, dos quais 23 do sexo masculino e 5 do feminino. Dos primeiros, dezenove eram adultos, três menores e uma ainda criança de 5 ou 6 anos de idade"(25)

Essa proporção entre homens e mulheres se mantém nas formas multibacilares. Alguns autores creditam o fato de que o homem trabalhando fora de casa estaria mais exposto ao contágio $^{(26)}$. No entanto, a proporção entre os sexos se igual ou nas formas paucibacilares.

Quanto à procedência, neste estudo predominaram os pacientes da capital (55,9\%) ou de outras áreas urbanas (73,6\%) do Estado do Acre, contrariamente ao que se encontrou, no vizinho Estado do Amazonas ${ }^{(14)}$. Esta diferença poderia ser explicada pela dificuldade de acesso da população ao serviço de referência, uma vez que a pesquisa não teve busca ativa (em loco) dos pacientes.

Nos grupos raciais, houve predomínio de mulatos e mesti- ços de índio, acompanhando a distribuição observada na população dessa região do país. No entanto, não foi observada associação de forma clínica com algum grupo racial, o mesmo já relatado por outros autores ${ }^{(20,24)}$.

A distribuição observada das formas clínicas tem semelhança com a de casuísticas ambulatoriais de outras localidades, sendo a virchowiana a mais freqüente com $43,8 \%{ }^{(14)}$, $52,3 \%{ }^{(21)}, 58,2 \%^{(9)}$ e $60 \%{ }^{(22)}$; e a indeterminada, a de menor ocorrência com 2,0\%(22) $3,9 \%^{(21)}, 7,9 \%^{(14)}$ e $9,0 \%{ }^{(9)}$.

Algumas características da hanseníase observadas neste estudo (média de idade de início da doença e duração da doença) foram mais elevadas do que as observadas no Estado do Amazonas ${ }^{(14)}$ e no Distrito Federal ${ }^{(21)}$. Não obstante, essas diferenças podem decorrer da abordagem metodológica. Neste trabalho, aquelas características foram extraídas da anamnese, enquanto outro, por exemplo, considerou a data da notificação compulsória ${ }^{(14)}$.

Houve predominância do uso da poliquimioterapia (PQT), como esquema terapêutico da hanseníase, embora 10,2\% tenham feito uso apenas da monoterapia que vigorou no Acre até 1986. Quanto à fase do tratamento, os pacientes com tratamento específico concluído foram maioria $(57,1 \%)$, ao contrário de outras pesquisas similares ${ }^{(14,21)}$, possivelmente porque neste serviço, além dos que tiveram a doença recémdiagnosticada, sejam avaliados também pacientes com tratamento concluído, pacientes com seqüelas em órgãos diversos ou problemas sociais.

Um dado preocupante foi o elevado índice (35/240) de recidiva da hanseníase, o qual é difícil de ser comparado com informações da literatura, que descreve freqüências de 1,09\% em casos paucibacilares e de $0,74 \%$ entre pacientes multibacilares seguidos por nove anos ${ }^{(24)}$. Por isto, é necessário estudo caso-controle para avaliar quais os fatores de risco para ocorrência deste evento em pessoas afetadas pela hanseníase e, desse modo, contribuir na prevenção do mesmo.

Para dimensionar a gravidade das alterações oculares da hanseníase potencialmente de risco para a visão, o melhor indicador seria a medida da acuidade visual. No entanto, não há uniformidade na conceituação de cegueira: visão menor que 0,1 ou $20 / 200$ para uns ${ }^{(27)}$, menor que 0,01 ou $20 / 400$ para outros $^{(28-29)}$. Anotações sobre acuidade visual podem levar a interpretações diferentes e até opostas ${ }^{(30)}$. Neste estudo, a cegueira foi considerada como visão menor que 0,1 ou 20/200 no olho com melhor acuidade, que é o conceito de cegueira legal no Brasil ${ }^{(31-32)}$

Assim, a freqüência observada de cegueira de $2 \%$ foi menor do que a encontrada no Distrito Federal $(2,6 \%)^{(21)}$; no Estado do Amazonas (9,7\%) $)^{(14)}$ e na cidade de Jundiaí $(3,6 \%)$, São Paulo ${ }^{(9)}$. Análise de vários estudos encontrou freqüência de visão menor que 20/200 em 5,4\% dos 4.772 pacientes afetados pela hanseníase e procedentes de todos os continentes $^{(28)}$. Outras pesquisas ${ }^{(33-35)}$ apontaram cegueira entre $3 \%$ a $10 \%$ dos pacientes. Portanto, neste corte, apesar do elevado número de pacientes de hanseníase com envolvimento ocular, a prevalência de cegueira pode ser considerada baixa. 
Todos os casos de cegueira observados nesta pesquisa foram em pacientes com tratamento para hanseníase concluído, logo, com maior tempo de doença. Muito embora não tenha havido associação significante de boa visão com alguma das formas clínicas, os pacientes cegos eram do grupo multibacilar.

As complicações da hanseníase envolvendo anexos do aparelho visual têm também interesse por causa do efeito antiestético da perda dos pêlos (madarose), estigmatizante da doença, além de indiretamente comprometer a função visual ao deixar a córnea vulnerável. Neste estudo, a madarose de cílios (20,5\%) e supercílios (16,1\%) foi a mais freqüente alteração dos anexos, à semelhança de outras pesquisas ${ }^{(10,36)}$.

Diagnosticada em 9,4\% dos pacientes, a hipotrofia dos músculos orbiculares é relevante porque se tratava da lesão precursora do lagoftalmo ${ }^{(1)}$. Essa hipotrofia pode levar à redundância da pele (dermatocálase) que, em outra investigação ${ }^{(21)}$, foi notada em $19 \%$ dos pacientes e que, parece, foi classificada por outro ${ }^{(14)}$ como ptose de cílios em 13,5\% dos casos.

Neste estudo, foi estatisticamente significante a associação de madarose e manchas palpebrais ao grupo multibacilar, bem como de madarose e hipotrofia palpebral com o grupo em tratamento concluído. Porém, no grupo em tratamento se associaram o eritema nodoso e as manchas palpebrais e conjuntivais. Estas manchas, possivelmente, foram agravadas pela clofazemina, usada no esquema de tratamento da forma multibacilar, cujos efeitos colaterais incluem pigmentação marrom da pele e conjuntiva ${ }^{(37)}$.

A diminuição da produção lacrimal (hipolacrimejamento), consistentemente encontrada entre as complicações do mal de Hansen $^{(13,38-39)}$, nesta casuística ocorreu em $14,2 \%$ dos pacientes. Ou seja, menos do que se anotou em $62 \%$ de 150 indivíduos $^{(39)}$; e mais do que se observou em 4,1\% de 218 pacientes $^{(11)}$.

Dentre as lesões ou complicações oculares propriamente ditas, destaca-se a diminuição da sensibilidade ou hipoestesia corneana de pelo menos um dos olhos em $27,6 \%$ dos pacientes, sendo também muito freqüente na maioria dos estudos publicados $^{(40)}$.

Nesta casuística, foi significativa a associação entre hipoestesia e ceratite ponteada, as quais predispõem ao maior sofrimento do epitélio da córnea e, consequentemente, às lesões mais graves, como as ulcerações. Contudo, não houve associação estatística entre hipoestesia e diminuição da secreção lacrimal.

Confirmando a expectativa, a catarata esteve presente nos pacientes com maior comprometimento da acuidade visual, sendo a segunda complicação de globo ocular mais diagnosticada neste estudo. O mesmo ocorre na maior parte dos estudos, exceto quando realizados em pacientes de hanseníase recém-diagnosticados ${ }^{(12)}$. A cegueira causada pela catarata preocupa, porque, apesar de reversível, a acuidade visual após cirurgia pode ser menor nos pacientes de hanseníase do que na população em geral, segundo alguns autores ${ }^{(41)}$. Ainda de acordo com os mesmos, esses pacientes cursam com processo inflamatório mais intenso no pós-operatório.
A usual terapêutica "generosa” de corticosteróides, como droga auxiliar no tratamento da hanseníase (dado aqui não analisado), pode ter contribuído para a menor prevalência de uveíte $^{(42)}$, outrora responsável por graves seqüelas oculares (Choyce apud) ${ }^{(43)}$. Porém a uveíte não deve ser negligenciada, considerando que 5 dos 6 casos deste estudo estavam assintomáticos.

Os sinais como atrofia de íris e pupila miótica foram seqüelas observadas nos pacientes que já estavam com tratamento específico concluído, com a hanseníase fora de atividade e com maior tempo de doença. Essas alterações irianas podem ter sido causadas pela “denervação” das estruturas (Oréfice apud) ${ }^{(44)}$ As lesões cicatriciais de coriorretinite, geralmente com baixa freqüência nessas populações, não são consideradas como decorrentes da hanseníase ${ }^{(1)}$.

A regressão logística aplicada neste estudo indicou que a idade acima de 40 anos e as formas multibacilares foram as mais determinantes na ocorrência das alterações oculares na hanseníase.

\section{CONCLUSÕES}

Algumas das pessoas (25,6\%) examinadas não manifestavam anormalidade ocular imputada à hanseníase, enquanto a maioria $(74,4 \%)$ era portadora de uma ou mais alterações. Estabelecido um critério de escores, observou-se que algumas variáveis apresentavam forte associação com a morbidade ocular, especialmente a idade acima de 40 anos e as formas multibacilares. Excluindo-se a madarose, a prevalência da morbidade ocular pelo $M$. leprae manteve-se elevada (48,4\%) nos pacientes estudados em ambulatório. Por sua vez, o conjunto dessas alterações oculares causa ou, potencialmente, pode causar limitações à qualidade de vida das pessoas afetadas pela hanseníase.

Campanhas devem ser feitas para que se detecte e se inicie o tratamento precocemente da doença, evitando-se assim a incapacidade visual ou alterações estigmatizantes. Especial atenção deve ser dada ao acompanhamento ocular de todos os casos de hanseníase, principalmente aqueles maiores de 40 anos de idade e/ou portadores de forma clínica multibacilar.

\section{ABSTRACT}

Purpose: To determine the prevalence of eye lesions in patients with active and non active leprosy who are being accompanied in a reference center. Methods: The transverse study consisted of ocular examinations and the study of demographic and clinical-epidemiological data, in the reference center in the city of Rio Branco, AC (Brazil). The study was conducted between October of 2001 and April of 2002. Results: Two hundred fifty-four patients were examined, the average age was 41.9 years, $70.1 \%$ were males and $29.9 \%$ females; $73.6 \%$ were from the urban area, $76.8 \%$ were of the 
764 Alterações oculares na hanseníase, observadas em pacientes ambulatoriais do serviço de referência da cidade de Rio Branco, Acre - Brasil

multibacillary form and $23.2 \%$ were of the paucibacillary form. Abnormalities of the ocular annexes were observed in $49.6 \%$ of the patients $(n=126)$ and of the ocular globe in $39.4 \%$ $(n=100)$, the most frequent being hypoesthesia of the cornea, cataract, madarosis, hiperchromic patches, hypolacrimation, and punctate keratitis. The eye lesions (74.4\%) had unequal frequencies $(\rho<0.0001)$, being predominant among those older than 40 years ( $48.4 \%$, against $26.0 \%$ in those under 40 years of age), multibacillary form (76.8\% against $23.2 \%$ in the paucibacillary form), and in those with a leprosy history of more than 5 years (76.8\% against 23.2\% leprosy history of less than 5 years). Ocular globe alterations were more frequent in patients who had concluded specific treatments $(\rho<0.05)$. Conclusions: The frequency of ocular morbidity in these leprosy patients, in almost three quarters (74.4\%) of those examined in the outpatient clinics, appeared similar to that described in literature and was more frequent in the multibacillary form of the disease and in patients over 40 years of age.

Keywords: Leprosy/complications; Eye infections, bacterial/ etiology Leprosy/epidemiology; Blindness/etiology; Brazil; Cross-sectional studies

\section{REFERÊNCIAS}

1. Campos WR, Rodrigues CAF, Oréfice F. Hanseníase. In: Oréfice F. editor. Uveíte: clínica \& cirúrgica: atlas \& texto. Rio de Janeiro: Cultura Médica; 2000. 341-66.

2. World Health Organization. Hanseniase. Weekly Epidemiol Record [cited 2002 Jan 4] 2002;77: [8 screens]. Available from URL: http://www.who.int/wer

3. Pan American Health Organization. Global alliance meets on elimination of leprosy [online] [cited 2000 April 14] 2002; [2 screens]. Available from: URL: http://www.paho.org/English/DPI/p020130.htm

4. Acre. Secretaria de Saúde do Estado do Acre. SESACRE. Programa de Dermatologia Sanitária da Secretaria de Saúde do Estado do Acre. Rondonia; SESACRE; 2001. [Mimeografado].

5. Cohen JM. Oftalmopatias tropicais. Arq Bras Oftalmol 1998;61:371-6.

6. Soshamma G, Suryawanshi N. Eye lesions in leprosy [commented on Lepr Ver 1989;60:328]. Lepr Rev 1989;60:33-8.

7. Johnstone PA, George AD, Meyers WM. Ocular lesions in leprosy. Ann Ophthalmol 1991;23:297-303.

8. World Health Organization. Data on visual impairment. Blindness and visual disability: other leading causes worldwide [online] [cited 2002 July 6] Available from URL:www.who.int/pbd/pbl/data.htm

9. Oliveira Neto HL, Silva JLM, Manso PG, Botene IM, Sartori MBF. Envolvimento ocular na hanseníase: estudo em pacientes de ambulatório. Arq Bras Oftalmol 1996;59:162-70.

10. Graff CC, Maia M, Arrata KM, Vicente LS, Arana J. Importância do exame oftalmológico precoce em portadores de hanseníase. Rev Bras Oftalmol 1999; 58:115-20.

11. Mvogo CE, Bella-Hiag AL, Ellong A, Achu JH, Nkeng PF. Ocular complications of leprosy in Cameroon. Acta Ophthalmol Scand 2001;79:31-3.

12. Çakiner T, Karçonlu MA. Ophthalmic findings of newly diagnosed leprosy patients in Istambul Leprosy Hospital, Turkey. Acta Ophthalmol Scand 1998; 76:100-2.

13. Monteiro LG, Campos WR, Oréfice F, Grossi MAF. Estudo das alterações oculares em hansenianos de controle ambulatorial. Rev Bras Oftalmol 1992; 51:167-70.

14. Cohen JM. Estudo epidemiológico das alterações oculares em hansenianos no Amazonas [tese]. Ribeirão Preto: Universidade de São Paulo; 1996.

15. Brasil. Ministério da Saúde. Normas técnicas para a eliminação da hanseníase no Brasil. Área Técnica de Dermatologia Sanitária - Brasília: Ministério da Saúde; 2001.
16. Brasil. Ministério da Saúde. Fundação Nacional da Saúde. Hanseníase. In: Fundação Nacional da Saúde. Guia de vigilância epidemiológica. [monografia online] [11 telas]. [citado 2002 Set 19]. Disponível em: URL:www.funasa.gov.br/ bup/GVE/GVE001.htm

17. Courtright PD. Defining the magnitude of ocular complications from Leprosy: problems of methodology. Int J Lepr Other Mycobact Dis 1988;56:566-72.

18. Moreno RD, Woods WJ. Prevalência das alterações oculares em pacientes portadores de hanseníase em um hospital colônia no Acre. Arq Bras Oftalmol 1999;62:167-70.

19. Costa MS, Gallo MEN, Nery JAC, Benchimol E. Avaliação oftalmológica em hanseníase multibacilar. Arq Bras Oftalmol 1999;62:701-3.

20. Margarido-Marchese L, Tedesco-Marchese AJ, Rivitti EA. Hanseníase. In Veronesi R, Focaccia R editores. Tratado de Infectologia. São Paulo: Atheneu; 1996. p.714-35.

21. Toríbio RC, Alvarez RRA, Mendes GF, de Souza ALB. Alterações oculares e incapacidade visual em pacientes com hanseníase - um estudo no Distrito Federal. An Bras Dermatol 2001;76:543-50.

22. Dana MR, Hocmman MA, Viana MAG, Hill CH, Sugar J. Ocular manifestations of leprosy in a noninstitutionalized community in the United States. Arch Ophthalmol 1994;112:626-9.

23. Ffytche TJ. Hansen's warning. Trans Med Soc Lond 1999;116:72-6.

24. Sridharan R, Lorenzo N. Leprosy. EMedicine Continuing Educ [serial online] [cited 2001 Dec 25]; 2: [30 telas]. Available from URL: http://www. emedicine.com/neuro/topic187.htm

25. Silva MR. Cultura e experiência sociais de hansenianos acreanos nas lutas contra a doença, os preconceitos e a exclusão social: Rio Branco e Sena Madureira (1930-1982). [dissertação]. Recife: Universidade Federal de Pernambuco/Universidade Federal do Acre; 2001.

26. Maradei J, Santos PM, Santos RCR, Olivalves SMR, Abreu MT. Complicações oculares como causa de incapacidade visual na hanseníase. Arq Bras Oftalmol 1998;61:11-4.

27. Lewallen S, Tungpakorn NC, Kim S, Courtright P. Progression of eye disease in "cured" leprosy patients: implications for understanding the pathophysiology of ocular disease and for addressing eyecare needs. $\mathrm{Br} \mathrm{J}$ Ophthalmol 2000;84:817-21.

28. Ffytche TJ. The prevalence of disabling ocular complication of leprosy: a global study. Indian J Lepr 1998;70:49-59.

29. Sundar Rao PSS, Daniel E, Kurian N, Gayathri KJ. Epidemiological aspects of ocular morbidity in leprosy-affected persons. Indian J Lepr 1998;70:115-22.

30. Bicas HEA. Acuidade visual. Medidas e anotações. Arq Bras Oftalmol 2002; 65:375-84

31. Conde AJM. Definindo a cegueira e a visão subnormal. [online] Instituto Benjamin Constant.[citado 2002 Jul 13]. Disponível em URL: http://www. ibcnet.org.br/Paginas/cegueira/Artigo_03.htm

32. Boni D, Moraes NSB, Freitas D. Evolução da acuidade visual em pacientes vítimas de trauma ocular por violência. Arq Brás Oftalmol [periódico online] 1997;62(1):1[citado 2002 Ago 6]. Disponível em: URL: www.abonet.com.br/ abo/abo621.htm

33. Malu KN, Malu AO. Blindness in leprosy patients of Kabuna State, Northern Nigéria. Trop Doct 1995;25:181-3.

34. Thompson K. Ocular morbidity in a sample of 150 treated leprosy patients. Indian J Lepr 1998;70:127-30.

35. Hogeweg M. Ocular leprosy. Int J Lepr Other Mycobact Dis 2001;69:530-5

36. Nwosu SNN, Nwosu MC. Ocular findings in leprosy patients in Nigeria. East Afr Med J 1994;71:441-4.

37. Kaur I, Ram J, Kumar B, Kaur S, Sharma VK. Effect of clofazimine on eye in multibacillary leprosy. Indian J Lepr 1990;62:87-90.

38. Piccinin MR, Conciani PA, Freitas AC, Tibana LA, Ferreira EL. Alterações oftalmológicas em pacientes hansenianos do Hospital São Julião. Rev Bras Oftalmol 2001;60:861-71.

39. Passerotti S, Salotti RA, Vieth H. Assessment and treatment of the dry eye in leprosy. Indian J Lepr 1998;70:103-8

40. Ocular complications of leprosy [editorial]. Lancet 1992;340:642-3.

41. Almeida EF, Almeida LNF. O uso de lentes intra-oculares em pacientes portadores de hanseníase. Arq Bras Oftalmol 2001;64:541-3.

42. Moreno RD. Alterações oculares na hanseníase, observadas em pacientes ambulatoriais da cidade de Rio Branco (Acre). [dissertação]. Rio Branco: Faculdade de Medicina da Universidade Federal da Bahia; 2002.

43. Job CK, Ebenezer GJ, Thompson K, Daniel E. Pathology of eye in leprosy. Indian J Lepr 1998;70:79-91.

44. Campos WR, Oréfice F, Sucena MA, Rodrigues CAF. Bilateral iridocyclitis caused by Micobacterium leprae diagnosed through paracentesis. Indian J Lepr 1998;70:27-31. 